\title{
TITLE:
}

\section{$<$ News> Report Meeting Ad-hoc Committee of IPS on the Great Ape Declaration with UNESCO}

AUTHOR(S):

Hooff, Jan van

\section{CITATION:}

Hooff, Jan van. <News> Report Meeting Ad-hoc Committee of IPS on

the Great Ape Declaration with UNESCO. Pan Africa News 2001, 8(2): 21 -

23

\section{ISSUE DATE:}

2001-12

URL:

http://hdl.handle.net/2433/143401

RIGHT:

Copyright (C) Pan Africa News. 


\section{<NEWS>}

In the evening of Oct.10, 2001, six members of the IPS ad-hoc committee for the great ape conservation and its legal advisor met at Paris, and the next day visited the World Heritage Center of UNESCO in order to discuss the feasibility of the World Heritage Species Concept and the possible methods of its implementation. Here, Dr. van Hooff summarized issues discussed in the meeting. This is a sequel to the draft proposal of the Great Ape Declaration published in the previous issue of $P A N(\mathrm{Ed}$.). 
Report Meeting Ad-hoc Committee of IPS on the Great Ape Declaration with UNESCO

\author{
Paris Oct. 11, 2001 \\ Participants: \\ On behalf of UNESCO World Heritage Center \\ Dr. Natarajan Ishwaran \\ (Chief, Natural Heritage Section) \\ Dr. Mario Hernandez \\ (Inform.Manager, Nat. Her. Sect.) \\ Dr. Joseph Mankoto Ma Mbaelele \\ (Progr. Homme et Biosphere) \\ On behalf of IPS \\ Dr. Toshisada Nishida (chairman Ad-hoc Comm.) \\ Dr. Vernon Reynolds (member) \\ Dr. Ian Redmond (member \& GRASP Initiative) \\ Dr. Christoph Boesch (member) \\ Dr. Richard Wrangham (member) \\ Dr. Steve Wise (legal adviser) \\ Dr. Jan van Hooff (secretray general; IPS)
}

The former president of the International Primatological Society, Dr. Toshisada Nishida has launched the initiative to acquire a special status. During a meeting with Dr. Ishwaran and Dr. Hernandez the following issues were discussed: The Concept of World Heritage Species. The proposition is to award a special protective status to "species of outstanding universal value from a scientific, education and cultural perspective, warranting a special conservation effort". This special status is sought, in the first place, for the Great Apes, namely because these are a "treasure of nature" of extreme value.

The arguments to select these species are:

- Their threatened status in the wild and increasingly rapid decline;

- Their close phylogenetic relationship with the Human species, since they are its relatives in the Animal kingdom;

-. Their highly developed cognitive and emotional abilities.

The new initiative is developed because it is felt that the existing treaties such as the CITES treaty and the Convention on Biodiversity offer insufficient protection. The CITES treaty, for instance, is restricted to international trade in endangered species and is not applicable at the within-nation level.
In most habitat countries there are laws concerning the treatment of protected species. However, law enforcement is often inconsistent and far from effective.

Under the existing UNESCO Convention on World Heritage Sites about 600 sites enjoy special protection because of an outstanding cultural ( \pm 500 sites) or natural value ( \pm 100 sites) for the world community. A new concept is being developed, namely of cultural landscapes (e.g. a volcano which has a special significance for a certain culture).

The natural sites cover important but limited parts of the great ape distribution. At the moment the WH Division is already engaged in developing a new framework which allows countries to form larger clusters of sites; such a site could cover a habitat range and be situated in more than one country. These could be made to cover major portions of the great ape distribution. An example is the World Heritage Africa Forest Initiative which is being developed at the moment in cooperation with WWF; this institution will also deliver a contribution matching that of UN Fund.

There are several possibilities to achieve the proposed special status for the Great Apes:

A) Amend the existing Convention on World Heritage Sites. The discussion revealed that an amendment of the existing convention may not be the most suitable for our purpose, since:

- A WH Species concept is of a comparatively different nature, and appears not to fit easily in the WH Sites convention.

- Countries that have ratified the existing Convention may not agree with the amendment and therefore pull out altogether.

.. Other countries may see this as an opportunity to bring in more amendments. The whole affair may thus be considerably retarded and even get out of control.

B) A second option may be to accommodate the "species concept" within the framework of the present WH Sites convention by adapting the operational guidelines. This might meet fewer problems. However, a main disadvantage would be that the operation would not yield the desired "splash", i.e. that it would not transmit effectively 
the sense of high urgency for protection of these species for which the community of nations accepts a special responsibility.

C) A third option is to work towards a completely new treaty within the WH framework: a World Heritage Species Treaty. It could then be developed specifically for the desired purpose. This will offer greater flexibility, and there will be no constraints of "entrenched compromises", which are at the base of the earlier treaties. The procedure will take up to 5 years (which may turn out to be not much longer than option $\mathrm{A}$ would take). The World Heritage treaties are conventions between UNESCO and the nations involved, and, indirectly, among those nations, since the relationships between nations should be in harmony with the general model.

The committee became aware (as far as it was not already) that the procedure is complex and requires extensive research concerning the legal and political aspects in order to bring a proposal in accordance with the required UNESCO format, legal formalisms and political ideologies. The committee has underestimated the complexity and the time schedule of the procedure.

The committee nevertheless agreed to go ahead. The WH Division of UNESCO has promised to provide all necessary advice in order to come to a proposal which has good prospects.

The following actions will be undertaken: A proposal will be made which combines the idea of a taxon oriented approach (initially for the great apes, but expandable to other "important", culturally significant taxa) and the "sites cluster" idea. The proposal will consist of three parts

a) The scientific argument: This part is in a near to final version. The final version will be drawn up as soon the research into the legal and political aspects has proceeded sufficiently to be incorporated with the scientific argument.

b) A legal and, c) a political part: Two legal experts have offered to research the conditions which a proposal should meet and to formulate these parts. They will first investigate whether a proposal to UNESCO is the most appropriate way to go, or whether there are other alternatives which might be more effectual and/or might require less time (Note that UNESCO is formally not part of the United Nations Organization; memberships are not identical: the USA pulled out of UNESCO years ago).

The experts are Gali Lynn HAGEL and Steve WISE: Gali HAGEL is a lawyer from Atlanta, USA, with relevant experience; she wrote and submitted a provisional document for the GAD ad-hoc committee, evaluating the strengths and weaknesses of the current approach in terms of what needs to be done for a formal approach to UNESCO, considering legal and practical aspects. Steve WISE is professor of law at Harvard University and author of "Rattling the Cage", a book arguing for raising the moral and legal status of the great apes. They expect to obtain funding for their part in the project (estimated at USD 75.000.-) from the GLASER Foundation and elsewhere. Their report intended to be the basis for an international workshop with legal experts, scheduled for late 2002. Since this is also the time when UNESCO celebrates its $30^{\text {th }}$ anniversary, this could be used to direct attention to the issue. The results of all of this should then be integrated in a definitive World Heritage Species proposal to be submitted jointly by UNESCO and UNEP to UN Fund. The United Nations Environmental Program is at present involved in the GRASP initiative (the Great Ape Survival Plan), namely a proposal for a UN Special Envoy for Great Apes and the development of a Global Strategy for Great Ape Conservation. The WHS-proposal should, therefore, be ready before May 2003 when the GRASP conference will be held.

(Reported by Jan van Hooff) 